\title{
Even Graphs
}

\section{F. Göbel}

H. J. Veldman

TWENTE UNIVERSITY OF TECHNOLOGY ENSCHEDE, THE NETHERLANDS

\section{ABSTRACT}

A nontrivial connected graph $G$ is called even if for each vertex $v$ of $G$ there is a unique vertex $\bar{v}$ such that $d(v, \bar{v})=\operatorname{diam} G$. Special classes of even graphs are defined and compared to each other. In particular, an even graph $G$ is called symmetric if $d(u, v)+d(u, \bar{v})=$ diam $G$ for all $u, v \in V(G)$. Several properties of even and symmetric even graphs are stated. For an even graph of order $n$ and diameter $d$ other than an even cycle it is shown that $n \geq 3 d-1$ and conjectured that $n \geq 4 d-4$. This conjecture is proved for symmetric even graphs and it is shown that for each pair of integers $n, d$ with $n$ even, $d \geq 2$ and $n \geq 4 d-4$ there exists an even graph of order $n$ and diameter $d$. Several ways of constructing new even graphs from known ones are presented.

\section{INTRODUCTION}

We use [1] for basic terminology and notations.

A nontrivial connected graph $G$ is called even if for each vertex $v$ of $G$ there is a unique vertex $\bar{v}$, the buddy of $v$, such that $d(v, \bar{v})=\operatorname{diam} G$. Even graphs are referred to as diametrical graphs in [2] and as self-centered unique eccentric point graphs in [3]. If $v$ is a vertex of an even graph, then $\bar{v}=v$, so the following observation is clear (cf. [3, Proposition 2]).

Proposition 1. Every even graph has even order.

Some examples of even graphs are:

- the cycle $C_{2 k}$ of diameter $k(k \geq 2)$,

- the $k$-cube $Q_{k}$ of diameter $k(k \geq 1)$,

- the graph $\overline{k K_{2}}$ of diameter $2(k \geq 2)$,

- the graph $K^{-}(k, k)$, obtained from $K(k, k)$

by deleting a perfect matching, of diameter $3(k \geq 3)$,

- the prism $K_{2} \times C_{2 k}$ of diameter $k+1(k \geq 2)$,

- the antiprism $A_{4 k+2}$ defined by 


$$
V\left(A_{4 k+2}\right)=\left\{u_{0}, u_{1}, \ldots, u_{2 k}, v_{0}, v_{1}, \ldots, v_{2 k}\right\}
$$

and, with indices $\bmod (2 k+1)$,

$$
\begin{aligned}
E\left(A_{4 k+2}\right)= & \left\{u_{i} u_{i+1} \mid 0 \leq i \leq 2 k\right\} \cup\left\{v_{i} v_{i+1} \mid 0 \leq i \leq 2 k\right\} \\
& \cup\left\{u_{i} v_{i} \mid 0 \leq i \leq 2 k\right\} \cup\left\{u_{i} v_{i+1} \mid 0 \leq i \leq 2 k\right\}
\end{aligned}
$$

$(k \geq 1) . A_{4 k+2}$ has diameter $k+1 . A_{10}$ is depicted in Figure 1. Observe that $\bar{u}_{i}=v_{i+k+1}$ and $\bar{v}_{i}=u_{i+k}(0 \leq i \leq 2 k$, indices $\bmod (2 k+1))$.

The only even graph of diameter 1 is $K_{2}$, while in [3] the following result is obtained.

Proposition 2 (Parthasarathy and Nandakumar [3]). The even graphs of diameter 2 are exactly the graphs $\overline{k K_{2}}$ with $k \geq 2$.

Using results of Section 2 it is easily shown that $K_{2}, C_{4}, \overline{3 K_{2}}, C_{6}, \overline{4 K_{2}}, Q_{3}$, and $C_{8}$ are the only even graphs of order at most 8.

\section{PROPERTIES}

We start with some simple, but useful observations.

Proposition 3. If $u$ and $v$ are adjacent vertices of an even graph $G$ of diameter $d$, then $d(v, \bar{u})=d-1$.

Proof. Since $d(u, \bar{u})=d$ and $v$ is adjacent to $u, d(v, \bar{u}) \geq d-1$. Since $v$ is not the buddy of $\bar{u}, d(v, \bar{u}) \leq d-1$.

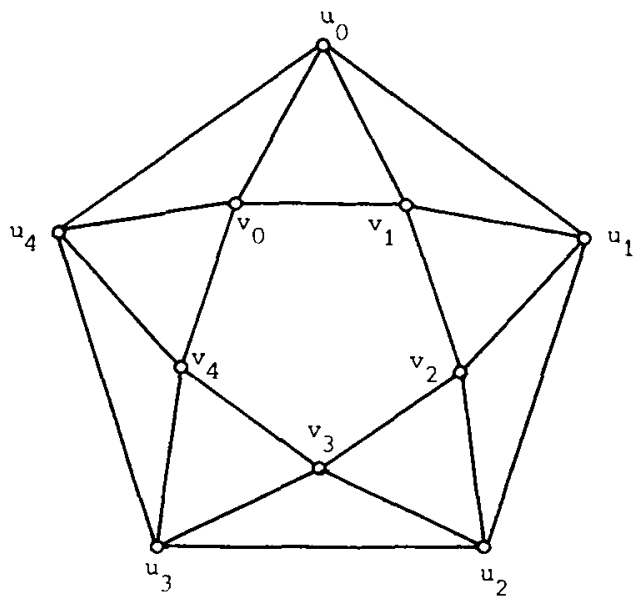

Fig. 1. The graph $\boldsymbol{A}_{10}$. 
If $u$ is a vertex of a graph $G$, then $\{v \in V(G) \mid d(u, v)=i\}$ is denoted by $N_{i}(u)$.

Proposition 4. Let $u$ and $v$ be vertices of an even graph $G$ of diameter $d$. If $v \in N_{i}(u)$ and $\bar{v} \in N_{j}(u)$, then $i+j \geq d$.

Proof. Suppose $i+j<d$. Then

$$
d(v, \bar{v}) \leq d(v, u)+d(u, \bar{v})=i+j<d,
$$

a contradiction.

Proposition 5. Let $u$ and $v$ be distinct vertices of an even graph $G$. If $P$ is a shortest $u-\bar{v}$ path and $Q$ a shortest $v-\bar{u}$ path in $G$, then $V(P) \cap V(Q)=\emptyset$.

Proof. Assuming the contrary, let $w$ be a common vertex of $P$ and $Q$. Since $u \neq v,|E(P)| \leq d-1$ and $|E(Q)| \leq d-1$, where $d=\operatorname{diam} G$. Hence

$$
|E(P)|+|E(Q)|=d(u, w)+d(w, \bar{v})+d(v, w)+d(w, \bar{u}) \leq 2 d-2 .
$$

Since $d(u, \bar{u}) \leq d(u, w)+d(w, \bar{u})$ and $d(v, \bar{v}) \leq d(v, w)+d(w, \bar{v})$, it follows that

$$
2 d=d(u, \bar{u})+d(v, \bar{v}) \leq 2 d-2,
$$

a contradiction.

A subset $S$ of the vertex set of a connected graph $G$ is called a vertex cut of $G$ if $G-S$ is disconnected.

Proposition 6. If $S$ is a vertex cut of an even graph $G$, then no vertex of $S$ has degree $|S|-1$ in the induced subgraph $\langle S\rangle$ of $G$.

Proof. Suppose $S$ contains a vertex $u$ with $\operatorname{deg}_{(S)} u=|S|-1$. Clearly $\bar{u} \notin S$. Let $G_{1}$ and $G_{2}$ be components of $G-S$ such that $\bar{u} \in V\left(G_{1}\right)$. Putting $d=\operatorname{diam} G$ we have $d(u, \bar{u})=d$ and, by Proposition $3, d(v, \bar{u})=d-1$ for each vertex $v$ of $S$ with $v \neq u$. Hence $d(w, \bar{u}) \geq d$ for each vertex $w$ of $G_{2}$, contradicting the uniqueness of $\bar{u}$.

Proposition 6 implies that no vertex cut of an even graph induces a complete subgraph. In particular, an even graph has no cut vertex.

Corollary 7. Every even graph other than $K_{2}$ is 2-connected.

The next result shows that even graphs of connectivity 2 necessarily contain a vertex of degree 2 . 
Theorem 8. If $\{u, v\}$ is a vertex cut of an even graph $G$, then $G-\{u, v\}$ has exactly two components, at least one of which contains only vertices of degree 2 in $G$.

Proof. Put $d=\operatorname{diam} G$. By Proposition 2 the only even graph of diameter 2 and connectivity 2 is $C_{4}$, so the theorem is true for $d=2$. We henceforth assume that $d \geq 3$ and distinguish two cases.

Case 1. $v=\bar{u}$

Case 1 is settled by showing that $G$ is an even cycle.

Let $G_{1}$ and $G_{2}$ be two components of $G-\{u, v\}, P_{1}: u, x_{1}, x_{2}, \ldots, x_{k}, v$ a shortest $u-v$ path in $\left\langle V\left(G_{1}\right) \cup\{u, v\}\right\rangle$ and $P_{2}: v, y_{1}, y_{2}, \ldots, y_{m}, u$ a shortest $v-u$ path in $\left\langle V\left(G_{2}\right) \cup\{u, v\}\right\rangle$. Since $d(u, v)=d$ and, by Proposition 3, $d\left(x_{1}, v\right)=$ $d\left(y_{1}, u\right)=d-1$, we have $k=m=d-1$. It follows that $y_{i}=\bar{x}_{i}(i=$ $1,2, \ldots, d-1)$. Since $\bar{x}_{1}$ is unique, $y_{1}$ is the only neighbor of $v$ outside $G_{1}$. Similarly, $x_{d-1}$ is the only neighbor of $v$ outside $G_{2}$. Thus deg $v=2$ and, symmetrically, $\operatorname{deg} u=2$. It follows that $G_{1}$ and $G_{2}$ are the only components of $G-\{u, v\}$.

Let $i$ be an integer with $1 \leq i \leq d-1$. To show that deg $x_{i}=2$, suppose $x_{i}$ has a neighbor $w \notin V\left(P_{1}\right)$. Let $P$ be a shortest $w-y_{i}$ path. Then $|E(P)| \leq d-1$ and $P$ contains exactly one of the vertices $u$ and $v$. Observe that $P$ necessarily contains $v$ if $i=1$ and $u$ if $i=d-1$. We proceed with the assumption that $P$ contains $u$, so that $i \neq 1$. Since $d\left(u, y_{i}\right)=d-i$, $d(w, u) \leq i-1$. Also, $d(w, u) \geq i-1$, otherwise

$$
d(u, v) \leq d(u, w)+d(w, v)<(i-1)+(d-i+1)=d .
$$

Hence $d(w, u)=i-1$. Since $d\left(w, y_{i-1}\right) \leq d-1$, it follows that a shortest $w-y_{i-1}$ path must contain $v$, so that $d(w, v) \leq d-i$. But then $d(u, v) \leq$ $d(u, w)+d(w, v) \leq d-1$, a contradiction. We conclude that $\operatorname{deg} x_{i}=2$, and symmetrically, $\operatorname{deg} y_{i}=2(i=1,2, \ldots, d-1)$, so that $G$ is an even cycle.

Case 2. $v \neq \bar{u}$.

Since $d(\bar{u}, \bar{v}) \leq d-1$, a shortest $\bar{u}-\bar{v}$ path contains neither $u$ nor $v$, implying that $\bar{u}$ and $\bar{v}$ are in the same component of $G-\{u, v\}$. Let $G_{1}$ be the component of $G-\{u, v\}$ containing $\bar{u}$ and $\bar{v}, G_{2}$ another component of $G-\{u, v\}$ and $P: u, x_{1}, x_{2}, \ldots, x_{k}, v$ a shortest $u-v$ path in $\left\langle V\left(G_{2}\right) \cup\{u, v\}\right\rangle$.

We first show that $\bar{x}_{1} \in V\left(G_{1}\right)$. By Proposition $3, d\left(u, \bar{x}_{1}\right)=d-1$. Assuming that $\bar{x}_{1} \notin V\left(G_{1}\right)$ we obtain

$$
\begin{aligned}
d\left(\bar{v}, \bar{x}_{1}\right) & =\min \left\{d(\bar{v}, u)+d\left(u, \bar{x}_{1}\right), d(\bar{v}, v)+d\left(v, \bar{x}_{1}\right)\right\} \\
& \geq \min \{1+(d-1), d+1\}=d,
\end{aligned}
$$

a contradiction. Hence $\bar{x}_{1} \in V\left(G_{1}\right)$. Next we show that $x_{1}$ is the only neighbor of $u$ outside $G_{1}$. Suppose $u$ has a neighbor $w \notin V\left(G_{1}\right)$. By Proposition 3, 
$d\left(x_{1}, \bar{u}\right)=d(w, \bar{u})=d-1$. A shortest $x_{1}-\bar{u}$ path cannot contain $u$, so $d\left(x_{1}, \bar{u}\right)=d\left(x_{1}, v\right)+d(v, \bar{u})$. Similarly, $d(w, \bar{u})=d(w, v)+d(v, \bar{u})$, implying that $d\left(x_{1}, v\right)=d(w, v)$. But then

$$
\begin{aligned}
d\left(\bar{x}_{1}, w\right) & =\min \left\{d\left(\bar{x}_{1}, u\right)+d(u, w), d\left(\bar{x}_{1}, v\right)+d(v, w)\right\} \\
& =\min \left\{d\left(\bar{x}_{1}, u\right)+d\left(u, x_{1}\right), d\left(\bar{x}_{1}, v\right)+d\left(v, x_{1}\right)\right\} \\
& =d\left(\bar{x}_{1}, x_{1}\right)=d
\end{aligned}
$$

contradicting the uniqueness of $\bar{x}_{1}$. Hence, indeed, $x_{1}$ is the only neighbor of $u$ outside $G_{1}$. Symmetrically, $x_{k}$ is the only neighbor of $v$ outside $G_{1}$. It follows that $G_{1}$ and $G_{2}$ are the only components of $G-\{u, v\}$. If $k=1$, then $V\left(G_{2}\right)=\left\{x_{1}\right\}$ and $\operatorname{deg} x_{1}=2$. If $k \geq 2$, then $\left\{x_{1}, v\right\}$ is again a vertex cut of $G$ with $v \neq \bar{x}_{1}$ and the above arguments apply to show that $x_{2}$ is the only neighbor of $x_{1}$ outside $V\left(G_{1}\right) \cup\{u\}$; hence $\operatorname{deg} x_{1}=2$. Via induction on $k$ it follows that $V\left(G_{2}\right)=\left\{x_{1}, x_{2}, \ldots, x_{k}\right\}$ and $\operatorname{deg} x_{1}=\operatorname{deg} x_{2}=\cdots=\operatorname{deg} x_{k}=2$.

Corollary 9. Every even graph $G$ with $\delta(G) \geq 3$ is 3 -connected.

Corollary 10. If $G$ is an even graph of order $n$ and diameter $d$ with $G \not \equiv C_{n}$, then $n \geq 3 d-1$.

Proof. The result is true if $d=1$. If $d \geq 2$, then $G$ contains a vertex $u$ with $\operatorname{deg} u \geq 3$. We show by contradiction that $\left|N_{i}(u)\right| \geq 3$ for $1 \leq i \leq$ $d-1$. Since $V(G)=\cup_{i=1}^{d-1} N_{i}(u) \cup\{u, \bar{u}\}$, it then follows that $n \geq 3(d-$ 1) $+2=3 d-1$.

Suppose $\left|N_{i}(u)\right|<3$ for some $i \in\{1,2, \ldots, d-1\}$. Since $N_{i}(u)$ is a vertex cut of $G$ and $G$ has no cut vertices, $\left|N_{i}(u)\right|=2$. Clearly, $i \neq 1$. From Proposition 4 we deduce that $\left\{\bar{v} \mid v \in N_{1}(u)\right\} \subseteq N_{d-1}(u)$, so that $\left|N_{d-1}(u)\right| \geq$ $\left|N_{1}(u)\right| \geq 3$ (cf. [3, Proposition 4]). Hence $i \neq d-1$. By Theorem 8, $G-N_{i}(u)$ has exactly two components, $\left\langle\cup_{j=0}^{i-1} N_{j}(u)\right\rangle$ and $\left\langle\cup_{j=i+1}^{d} N_{j}(u)\right\rangle$, one of which contains vertices of degree 2 only. Since $\cup_{j=0}^{i-l} N_{j}(u)$ contains the vertex $u$ of degree at least 3 , the vertices of $\cup_{j=i+1}^{d} N_{j}(u)$ all have degree 2 . It follows that $\left|N_{j}(u)\right|=2$ for $i+1 \leq j \leq d-1$. In particular $\left|N_{d-1}(u)\right|=2$, a contradiction.

Corollary 10 might be improved in the following way.

Conjecture 11. If $G$ is an even graph of order $n$ and diameter $d$ with $G \not$ $C_{n}$, then $n \geq 4 d-4$.

Corollary 10 implies that Conjecture 11 is true for $d \leq 4$. Using ad hoc arguments involving several results of the present section, we have verified it for $d \in\{5,6\}$. In Section 3 Conjecture 11 will be proved for a special class of even graphs. In Section 4 we shall prove that for each pair of integers $n, d$ with 
$n$ even, $d \geq 2$ and $n \geq 4 d-4$ there exists an even graph of order $n$ and diameter $d$, showing, in particular, that Conjecture 11 is best possible for $d \geq 2$.

\section{SPECIAL EVEN GRAPHS}

An even graph $G$ is called balanced if $\operatorname{deg} v=\operatorname{deg} \bar{v}$ for each $v \in V(G)$, harmonic if $\bar{u} \bar{v} \in E(G)$ whenever $u v \in E(G)$, and symmetric if $d(u, v)+$ $d(u, \bar{v})=\operatorname{diam} G$ for all $u, v \in V(G)$. Observe that an even graph $G$ is symmetric if and only if the inequality in Proposition 4 holds with equality for all $u, v \in V(G)$. An even graph $G$ is said to be maximal if there is no even graph $H$ such that $|V(H)|=|V(G)|$, $\operatorname{diam} H=\operatorname{diam} G$ and $G \varsubsetneqq H$. From this definition it follows that an even graph $G$ is maximal if and only if $G$ contains no pair $u, v$ of nonadjacent vertices such that $G+u v$ is an even graph of the same diameter as $G$. All examples of even graphs given in Section 1 are balanced, harmonic, symmetric and maximal. We establish some relations between the defined notions.

Proposition 12. Every harmonic even graph is balanced.

Proof. Let $u$ be a vertex of a harmonic even graph. Then $\{\bar{v} \mid v \in N(u)\} \subseteq$ $N(\bar{u})$.

Hence

$$
\operatorname{deg} u=|N(u)|=|\{\bar{v} \mid v \in N(u)\}| \leq|N(\bar{u})|=\operatorname{deg} \bar{u} .
$$

Also, $\operatorname{deg} \bar{u} \leq \operatorname{deg} \bar{u}=\operatorname{deg} u$.

Proposition 13. Every symmetric even graph is harmonic.

Proof. Let $G$ be a symmetric even graph and $u v$ an edge of $G$. Since $d(u, v)=1, d(\bar{u}, v)=d-1$. Hence $d(\bar{u}, \bar{v})=1$, or, equivalently, $\bar{u} \bar{v} \in$ $E(G)$.

Proposition 14. Every symmetric even graph is maximal.

Proof. Suppose there exists a symmetric even graph $G$ of diameter $d$ which is not maximal. Then $G$ contains two nonadjacent vertices $u$ and $v$ such that $G+u v$ is an even graph of diameter $d$ too. Since $d_{G}(u, v) \geq 2, d_{G}(v, \bar{u}) \leq$ $d-2$. But then

$$
d_{G+u v}(u, \bar{u}) \leq d_{G+u v}(u, v)+d_{G+u v}(v, \bar{u}) \leq 1+(d-2)=d-1,
$$

a contradiction. 
If $G$ is a harmonic even graph of diameter at least 2 , then the edges of $G$ occur in pairs $(u v, \bar{u} \bar{v})$ with $u v \neq \bar{u} \bar{v}$, implying the following result.

Proposition 15. Every harmonic even graph except $K_{2}$ has even size.

If $G$ is a nonmaximal even graph and $H$ a maximal even graph with $|V(H)|=|V(G)|, \operatorname{diam} H=\operatorname{diam} G$ and $G \subset H$, then every graph $G^{\prime}$ with $G \subset G^{\prime} \subset H$ is an even graph of the same diameter as $G$. Hence nonmaximal even graphs need not be balanced and need not have even size. The even graph $H_{1}$ in Figure 2, being a proper spanning subgraph of $K_{2} \times C_{6}$, is nonmaximal, not balanced and has odd size. In [3] the following result is proved.

Theorem 16 (Parthasarathy and Nandakumar [3]). Every maximal even graph of order $n$ and diameter 3 is $\frac{1}{2}(n-2)$-regular.

Maximal even graphs of diameter at least 4 need not be regular. The even graphs of Figure 3 in Section 4 are all symmetric, hence maximal, but nonregular. One might expect that maximal even graphs of diameter at least 4 are balanced. However, the even graph $\mathrm{H}_{2}$ in Figure 2 is maximal, but not balanced. Observe that $\mathrm{H}_{2}$ has odd size.

The graph $\mathrm{H}_{3}$ is an example of a harmonic even graph which is not maximal and hence not symmetric. The graph $H_{4}$ shows that a balanced even graph need not be harmonic and may have odd size.

The fact that the even graph $H_{3}$ is not symmetric is also immediate from the following result.

Theorem 17. If $G$ is a symmetric even graph, then $G$ is 3-connected unles $G \cong K_{2}$ or $G$ is an even cycle.

Proof. Let $G$ be a symmetric even graph of diameter $d \geq 2$ which is not 3 -connected. We show that $G$ is an even cycle. By Corollary $9, G$ contains a vertex $u$ of degree 2 . Let $u_{1}$ and $u_{2}$ be the neighbors of $u$. Then by Proposition $13 \bar{u}_{1}$ and $\bar{u}_{2}$ arè the neighbors of $\bar{u}$. Every shortest $u-\bar{u}$ path either contains $u_{1}$ and $\bar{u}_{2}$ or $u_{2}$ and $\bar{u}_{1}$. By definition of a symmetric even graph, each vertex of $G$ is on a shortest $u-\bar{u}$ path. Hence each vertex of $G-\{u, \bar{u}\}$ belongs to either a shortest $u_{1}-\bar{u}_{2}$ path or a shortest $u_{2}-\bar{u}_{1}$ path. For $i=1,2$, let $S_{i}$ be the set of all vertices of $G-\{u, \bar{u}\}$ which are on a shortest $u_{i}-\bar{u}_{3-i}$ path. By Proposition 5, $S_{1} \cap S_{2}=\emptyset$. Suppose there exists an edge $v_{1} v_{2} \in$ $E(G)$ with $v_{i} \in S_{i}(i=1,2)$ and assume without loss of generality that $d\left(u_{1}, v_{1}\right) \leq d\left(u_{2}, v_{2}\right)$. Then

$$
\begin{aligned}
d\left(u_{1}, \bar{u}_{1}\right) \leq d\left(u_{1}, v_{1}\right)+d( & \left(v_{1}, v_{2}\right)+d\left(v_{2}, \bar{u}_{1}\right)= \\
& d\left(u_{1}, v_{1}\right)+1+\left(d-2-d\left(u_{2}, v_{2}\right)\right) \leq d-1,
\end{aligned}
$$

a contradiction. Hence, in fact, $\left\{v_{1} v_{2} \in E(G) \mid v_{1} \in S_{1}, v_{2} \in S_{2}\right\}=\emptyset$. It follows that $\{u, \bar{u}\}$ is a vertex cut of $G$. By Theorem $8, S_{1}$ or $S_{2}, S_{1}$, say, consists 

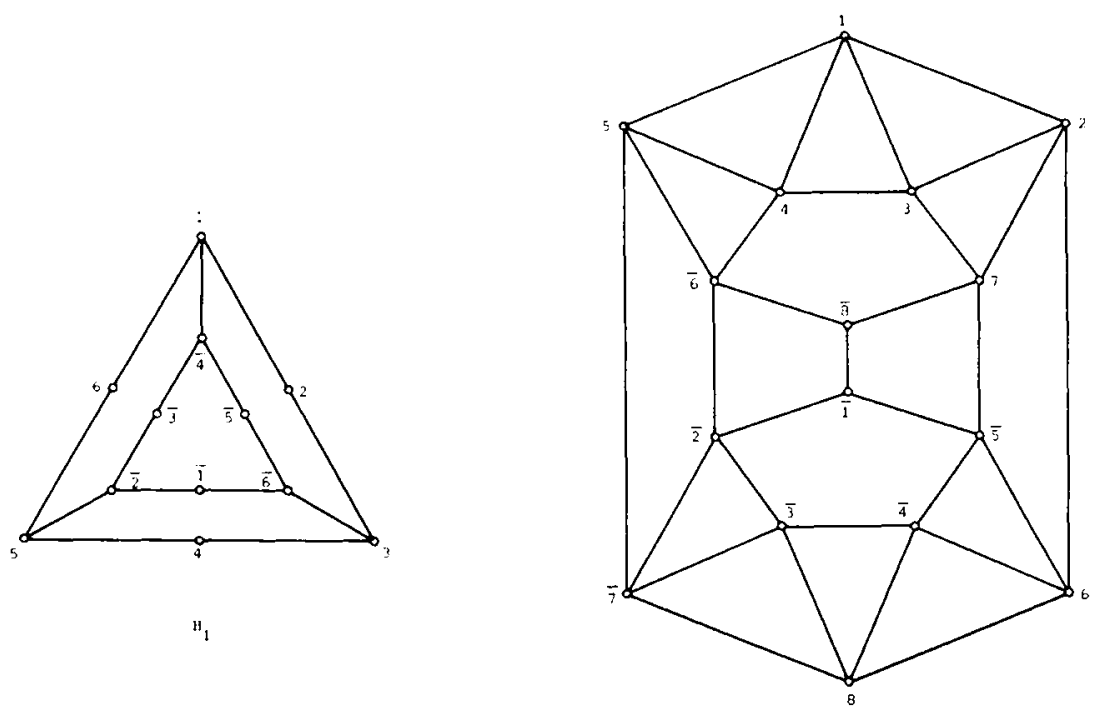

$\mathrm{H}_{2}$
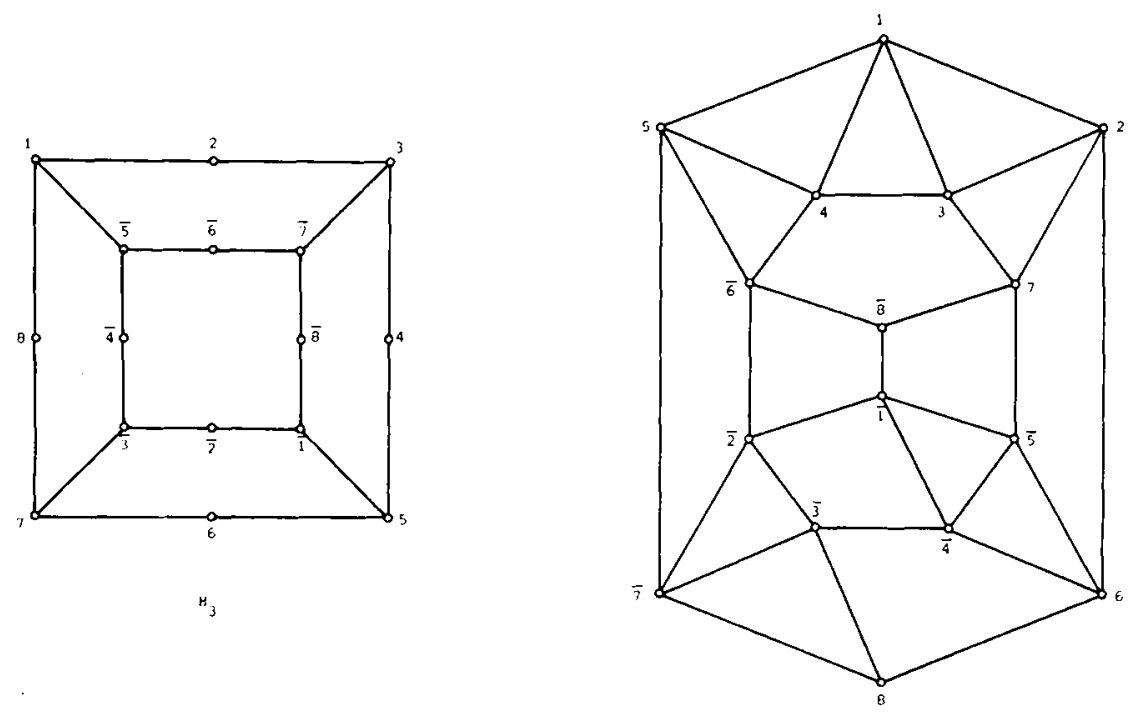

Fig. 2

of vertices of degree 2 only. Since each pair of vertices of $S_{i}$ has distance at most $d-2$, we have $\bar{v} \in S_{3-i}$ whenever $v \in S_{i}(i=1,2)$. Thus $S_{2}=\{\bar{v} \mid v \in$ $\left.S_{1}\right\}$. Since every symmetric graph is balanced, all vertices of $S_{2}$, too, have degree 2 . We conclude that $G$ is an even cycle. 
We now show that Conjecture 11 is true for symmetric even graphs.

Theorem 18. If $G$ is a symmetric even graph of order $n$ and diameter $d$ with $G \not \equiv C_{n}$, then $n \geq 4 d-4$.

Proof. For $d \leq 4$ the theorem is a consequence of Corollary 10; so assume $d \geq 5$. Let $u$ be a vertex of $G$. By Theorem 17, $\operatorname{deg} u \geq 3$. We show by contradiction that $\left|N_{i}(u)\right| \geq 4$ for $2 \leq i \leq d-2$, implying that

$$
\begin{aligned}
n & =|\{u, \bar{u}\}|+\left|N_{1}(u)\right|+\left|N_{d-1}(u)\right|+\left|\bigcup_{i=2}^{d-2} N_{i}(u)\right| \geq 2+3+3+4(d-3) \\
& =4 d-4 .
\end{aligned}
$$

Put $I=\left\{i|2 \leq i \leq d-2,| N_{i}(u) \mid<4\right\}$. Assuming that $I \neq \emptyset$, let $m$ be the smallest integer in $I$. Since $N_{m}(u)$ is a vertex cut of $G,\left|N_{m}(u)\right|=3$ by Theorem 17. The definition of a symmetric even graph implies that $N_{d-i}(u)=$ $\left\{\bar{v} \mid v \in N_{i}(u)\right\}$ for $0 \leq i \leq d$. In particular $\left|N_{i}(u)\right|=\left|N_{d-i}(u)\right|(0 \leq i \leq d)$ and, for even $d,\left|N_{d / 2}(u)\right|$ is even. We conclude that $m \leq \frac{1}{2}(d-1)$. Let $u_{1}, u_{2}, u_{3}$ be the vertices of $N_{m}(u)$. We now show by contradiction that each vertex of $N_{m}(u)$ has exactly one neighbor in $N_{m-1}(u)$.

Suppose, e.g., $u_{1}$ has two distinct neighbors $v_{1}$ and $v_{2}$ in $N_{m-1}(u)$. For $i=1,2$, let $P_{i}$ be a shortest $v_{i}-\bar{u}_{1}$ path in $G$. By Proposition 3, $\left|E\left(P_{i}\right)\right|=$ $d-1(i=1,2)$. Since $\bar{u}_{1} \in N_{d-m}(u)$ and $d-m>m, P_{i}$ contains a vertex of $N_{m}(u)(i=1,2)$. This vertex cannot be $u_{1}$, so $P_{i}$ contains $u_{2}$ or $u_{3}(i=1,2)$. Let $Q_{i}$ be a shortest $v_{i}-\bar{v}_{3-i}$ path in $G$, so that $\left|E\left(Q_{i}\right)\right| \leq d-1(i=1,2)$. Since $\bar{v}_{3-i} \in N_{d^{-m+1}}(u), Q_{i}$ contains a vertex of $N_{m}(u)(i=1,2) . Q_{i}$ does not contain $u_{1}$, otherwise $\left|E\left(Q_{i}\right)\right|>d\left(u_{1}, \bar{v}_{3-i}\right)=d-1(i=1,2)$. Hence $Q_{i}$, too, contains $u_{2}$ or $u_{3}(i=1,2)$. We now derive contradictions in all possible cases.

Case 1. $\quad P_{1}$ contains $u_{2}$ and $P_{2}$ contains $u_{3}$ (or, symmetrically, $P_{1}$ contains $u_{3}$ and $P_{2}$ contains $u_{2}$ ). By Proposition 5, $Q_{1}$ and $Q_{2}$ are disjoint. We distinguish two subcases.

Case $1.1 Q_{1}$ contains $u_{2}$ and $Q_{2}$ contains $u_{3}$. Since $\bar{u}_{1} \in N_{d-m}(u)$, $\min _{1 \leq i \leq 3}\left\{d\left(u_{i}, \bar{u}_{1}\right)\right\}=d-2 m$. Hence either $d\left(u_{2}, \bar{u}_{1}\right)=d-2 m$ or $d\left(u_{3}, \bar{u}_{1}\right)=d-2 m$. If $d\left(u_{2}, \bar{u}_{1}\right)=d-2 m$, then, since $d\left(u_{2}, \bar{v}_{2}\right) \geq$ $(d-m+1)-m$,

$$
\left|E\left(Q_{1}\right)\right|=d\left(v_{1}, u_{2}\right)+d\left(u_{2}, \bar{v}_{2}\right)>d\left(v_{1}, u_{2}\right)+d\left(u_{2}, \bar{u}_{1}\right)=\left|E\left(P_{1}\right)\right|=d-1,
$$

a contradiction. If $d\left(u_{3}, \bar{u}_{1}\right)=d-2 m$, then we obtain the analogous contradiction that $\left|E\left(Q_{2}\right)\right|>\left|E\left(P_{2}\right)\right|=d-1$.

Case 1.2. $Q_{1}$ contains $u_{3}$ and $Q_{2}$ contains $u_{2}$. Observing that

$$
d\left(v_{1}, u_{3}\right)+d\left(u_{3}, \bar{u}_{1}\right) \geq\left|E\left(P_{1}\right)\right|=\left|E\left(P_{2}\right)\right|=d\left(v_{2}, u_{3}\right)+d\left(u_{3}, \bar{u}_{1}\right),
$$


we conclude that $d\left(v_{1}, u_{3}\right) \geq d\left(v_{2}, u_{3}\right)$, thereby reaching the following contradiction:

$$
\left|E\left(Q_{1}\right)\right|=d\left(v_{1}, u_{3}\right)+d\left(u_{3}, \bar{v}_{2}\right) \geq d\left(v_{2}, u_{3}\right)+d\left(u_{3}, \bar{v}_{2}\right) \geq d\left(v_{2}, \bar{v}_{2}\right)=d
$$

Case 2. Both $P_{1}$ and $P_{2}$ contain $u_{2}$ (or, symmetrically, both $P_{1}$ and $P_{2}$ contain $\left.u_{3}\right)$. Then $d\left(v_{1}, u_{2}\right)=d\left(v_{2}, u_{2}\right)$. Assuming that $Q_{1}$ contains $u_{2}$, we obtain the following contradiction:

$$
\left|E\left(Q_{1}\right)\right|=d\left(v_{1}, u_{2}\right)+d\left(u_{2}, \bar{v}_{2}\right)=d\left(v_{2}, u_{2}\right)+d\left(u_{2}, \bar{v}_{2}\right) \geq d\left(v_{2}, \bar{v}_{2}\right)=d
$$

Hence $u_{2} \notin V\left(Q_{1}\right)$. Similarly, $u_{2} \notin V\left(Q_{2}\right)$. But then $u_{3} \in V\left(Q_{1}\right) \cap V\left(Q_{2}\right)$, contradicting Proposition 5.

The above contradictions show that, indeed, each vertex of $N_{m}(u)$ has exactly one neighbor in $N_{m-1}(u)$. On the other hand, each vertex of $N_{m-1}(u)$ has at least one neighbor in $N_{m}(u)$, since each vertex of $G$ is on a shortest $u-\bar{u}$ path. It follows that $\left|N_{m-1}(u)\right| \leq 3$. Since $m$ is the smallest integer in $I$, we conclude that $m=2$ and $\left|N_{1}(u)\right|=\operatorname{deg} u=3$, while each vertex of $N_{1}(u)$ has exactly one neighbor in $N_{2}(u)$. Hence, since $\delta(G) \geq 3$, each vertex of $N_{1}(u)$ has at least one neighbor in $N_{1}(u)$, implying that $\Delta\left(\left\langle N_{1}(u)\right\rangle\right)=2=\left|N_{1}(u)\right|-1$. This contradiction with Proposition 6 completes the proof.

For $d \geq 2$ the graph $K_{2} \times C_{2 d-2}$ is a symmetric even graph of diameter $d$ and order $4 d-4$, showing that Theorem 18 is best possible for $d \geq 2$.

\section{CONSTRUCTIONS}

We start with some results which can be used to construct new even graphs from known ones. The proofs are straightforward, using only the definitions of even and symmetric even graphs. On each result we give a brief comment, in which the buddies of the vertices of the new even graph and its diameter are given.

Proposition 19. The cartesian product $G \times H$ of two graphs $G$ and $H$ is even if and only if both $G$ and $H$ are even. Also, $G \times H$ is a symmetric even graph if and only if both $G$ and $H$ are symmetric even graphs.

Comment. If $G$ and $H$ are even and $(u, v) \in V(G \times H)$, then $(\overline{u, v})=$ $(\bar{u}, \bar{v}) . \operatorname{diam}(G \times H)=\operatorname{diam} G+\operatorname{diam} H$.

The edges of a cartesian product $G \times H$ fall into two classes in a natural way; we speak of $G$-edges and $H$-edges. 
Proposition 20. Let $G$ be an even graph of diameter at least 3 and $S$ an independent set of vertices of $G$. If $A$ is the set of $K_{2}$-edges of $K_{2} \times G$ incident with the vertices in the two copies of $S$, then $\left(K_{2} \times G\right)-A$ is even.

Comment. Each vertex of $\left(K_{2} \times G\right)-A$ has the same buddy as in $K_{2} \times G$. $\operatorname{diam}\left(K_{2} \times G\right)-A=\operatorname{diam} G+1$.

Let $G$ be a graph, $S$ a subset of $V(G)$, and $A$ the set of edges of $G$ incident with exactly one vertex of $S$. Then we define $P_{k}(G, S)$ as the graph obtained from $P_{k} \times G$ by deleting the edges of $A$ in each copy of $G$ corresponding to a vertex of $P_{k}$ of degree 2. Furthermore, $P_{k}(G)$ denotes the graph obtained from $P_{k} \times G$ by deleting all edges in each copy of $G$ corresponding to a vertex of $P_{k}$ of degree 2 . Alternatively, $P_{k}(G)$ is the graph obtained from $K_{2} \times G$ by subdividing each $K_{2}$-edge $k-1$ times.

Proposition 21. Let $G$ be an even graph and $S$ a subset of $V(G)$ such that $V(G)-S=\{\bar{v} \mid v \in S\}$. Then every graph $H$ with $P_{k}(G) \subset H \subset P_{k}(G, S)$ is even.

Comment. Let the vertices of $P_{k}$ be labeled $u_{1}, u_{2}, \ldots, u_{k}$ in the natural order. The buddy of $\left(u_{i}, v\right) \in V(H)=V\left(P_{k} \times G\right)$ is $\left(u_{k+1-i}, \bar{v}\right)(i=1,2, \ldots, k)$. $\operatorname{diam} H=\operatorname{diam} G+k-1$.

If $G$ is a connected graph, $u$ a vertex of $G$ and $S$ a subset of $V(G)$, then we denote $\min \{d(u, v) \mid v \in S\}$ by $d(u, S)$.

Proposition 22. Let $G$ be an even graph, $S$ a subset of $V(G)$ and $S^{\prime}=$ $\{u \mid u \in S\}$.

Assume that the following conditions are satisfied:

(i) $d(u, v) \leq 2$ whenever $u, v \in S$ or $u, v \in S^{\prime}$;

(ii) $d(u, S) \leq \operatorname{diam} G-2$ and $d\left(u, S^{\prime}\right) \leq \operatorname{diam} G-2$ for all $u \in V(G)$. Then the graph $H$ with $V(H)=V(G) \cup\{x, y\}$, where $x, y \notin V(G)$, and $E(H)=E(G) \cup\{x u \mid u \in S\} \cup\left\{y v \mid v \in S^{\prime}\right\}$ is even.

Comment. The vertices $x$ and $y$ are buddies in $H$, and each other vertex of $H$ has the same buddy as in $G$. diam $H=\operatorname{diam} G$.

As an example, Proposition 22 is applicable if $G$ is a symmetric even graph, $\langle S\rangle \cong C_{4}$ and no vertex of $G$ is adjacent to all vertices of $S$.

Proposition 23. Let $G$ be a symmetric even graph with $G \not \equiv K_{2}$. Then the line graph $L(G)$ is even if and only if $G$ contains no triangle. 
Comment. If $G$ is a symmetric even graph containing no triangle and $e$ the vertex of $L(G)$ corresponding to the edge $u v$ of $G$, then $\bar{e}$ is the vertex of $L(G)$ corresponding to the edge $\bar{u} \bar{v}$ of $G$ and $\operatorname{diam} L(G)=\operatorname{diam} G$.

If $G$ is a graph and $k$ an integer with $k \geq 1$, then we denote by $S_{k}(G)$ the graph obtained from $G$ by subdividing each edge $k-1$ times.

Proposition 24. Let $G$ be a symmetric even graph with $G \not \equiv K_{2}$ and $k$ an integer with $k \geq 2$. Then $S_{k}(G)$ is even if and only if $G$ contains no triangle.

Comment. Let $G$ be a symmetric even graph containing no triangle, and $P: u=u_{0}, u_{1}, \ldots, u_{k}=v$ and $Q: \bar{u}=v_{0}, v_{1}, \ldots, v_{k}=\bar{v}$, respectively, the paths of $S_{k}(G)$ obtained when the edges $u v$ and $\bar{u} v$ of $G$ are subdivided $k-1$ times. Then $\bar{u}_{i}=v_{i}(i=0,1, \ldots, k) . \operatorname{diam} S_{k}(G)=k \operatorname{diam} G$.

We define the truncation $\operatorname{Tr}(G)$ of a graph $G$ as the graph with vertex set $\{(u, v) \mid u v \in E(G)\}$ in which two vertices $\left(u_{1}, v_{1}\right)$ and $\left(u_{2}, v_{2}\right)$ are adjacent if and only if either $u_{1}=u_{2}$ or $u_{1}=v_{2}$ and $v_{1}=u_{2}$. Roughly speaking, $\operatorname{Tr}(G)$ is obtained from $G$ by replacing each vertex $v$ of $G$ by a complete graph of order $\operatorname{deg} v$.

Proposition 25. Let $G$ be a symmetric even graph. $\operatorname{Then} \operatorname{Tr}(G)$ is even if and only if $G$ contains no triangle.

Comment. If $G$ is a symmetric even graph containing no triangle and $(u, v) \in V(\operatorname{Tr}(G))$, then $(\overline{u, v})=(\bar{u}, \bar{v})$ and $\operatorname{diam} \operatorname{Tr}(G)=2 \operatorname{diam} G$.

Using the described constructions we finally prove the result announced at the end of Section 2.

Theorem 26. For each pair of integers $n, d$ with $n$ even, $d \geq 2$ and $n \geq 4 d-4$ there exists a harmonic even graph of order $n$ and diameter $d$.

Proof. We give a constructive proof by induction on $d$. For $d=2$ the assertion holds by Proposition 2. Now assume that $d \geq 3$ and there exist harmonic even graphs of order $n$ and diameter $d-1$ for each integer $n$ with $n \geq$ $4(d-1)-4=4 d-8$.

Let $G$ be a harmonic even graph of diameter $d-1$. Then $K_{2} \times G$ is a harmonic even graph of order $2|V(G)|$ and diameter $d$. If $S$ is the vertex set of a 4-cycle of $K_{2} \times G$ containing two $K_{2}$-edges, then, since $G$ is harmonic, the buddies of the vertices of $S$ are also on a 4-cycle of $K_{2} \times G$. Now Proposition 22 can be applied to $K_{2} \times G$ and $S$ in order to obtain a harmonic even graph of order $2|V(G)|+2$ and diameter $d$. From the induction hypothesis we conclude 
that harmonic even graphs of order $n$ and diameter $d$ exist for each even integer $n \geq 2(4 d-8)=8 d-16$.

We now construct a symmetric even graph $G_{n, d}$ of order $n$ and diameter $d$ for each even integer $n$ with $4 d-4 \leq n \leq 6 d-6$ and a symmetric even graph $H_{n, d}$ of order $n$ and diameter $d$ for each even integer $n$ with $6 d-8 \leq n \leq$ $8 d-14$. The proof is then complete, since every symmetric even graph is harmonic.

The graph $G_{n, d}$ is defined by

$$
\begin{aligned}
V\left(G_{n, d}\right)=\{ & u_{0}, u_{1}, \ldots, u_{2 d-3}, v_{0}, v_{1}, \ldots, v_{2 d-3}, \\
& \left.\cdot x_{0}, x_{1}, \ldots, x_{n / 2}-2 d+1, y_{0}, y_{1}, \ldots, y_{n 2-2 d+1}\right\}
\end{aligned}
$$

and, with indices $\bmod (2 d-2)$,

$$
\begin{aligned}
E\left(G_{n, d}\right)= & \left\{u_{i} u_{i+1} \mid 0 \leq i \leq 2 d-3\right\} \cup\left\{v_{i} v_{i+1} \mid 0 \leq i \leq 2 d-3\right\} \\
& \cup\left\{u_{i} v_{i} \mid 0 \leq i \leq 2 d-3\right\} \cup\left\{x_{i} u_{i} \mid 0 \leq i \leq \frac{1}{2} n-2 d+1\right\} \\
& \cup\left\{x_{i} u_{i+1} \mid 0 \leq i \leq \frac{1}{2} n-2 d+1\right\} \cup\left\{x_{i} v_{i} \mid 0 \leq i \leq \frac{1}{2} n-2 d+1\right\} \\
& \cup\left\{x_{i} v_{i+1} \mid 0 \leq i \leq \frac{1}{2} n-2 d+1\right\} \cup\left\{x_{i} x_{i+1} \mid 0 \leq i \leq \frac{1}{2} n-2 d\right\} \\
& \cup\left\{y_{i} u_{d-1+i} \mid 0 \leq i \leq \frac{1}{2} n-2 d+1\right\} \\
& \cup\left\{y_{i} u_{d+i} \mid 0 \leq i \leq \frac{1}{2} n-2 d+1\right\} \\
& \cup\left\{y_{i} v_{d-1+i} \mid 0 \leq i \leq \frac{1}{2} n-2 d+1\right\} \\
& \cup\left\{y_{i} v_{d+i} \mid 0 \leq i \leq \frac{1}{2} n-2 d+1\right\} \\
& \cup\left\{y_{i} y_{i+1} \mid 0 \leq i \leq \frac{1}{2} n-2 d\right\} .
\end{aligned}
$$

Observe that $G_{4 d-4, d} \cong K_{2} \times C_{2 d-2}$ and, for $n$ even and $4 d-4<n \leq$ $6 d-6$, the graph $G_{n, d}$ can be obtained from $K_{2} \times C_{2 d-2}$ by repeated application of Proposition 22.

The graph $H_{n, d}$ is obtained from the graph $G_{n-2 d+4, d}$ as follows:

$$
V\left(H_{n, d}\right)=V\left(G_{n-2 d+4, d}\right) \cup\left\{w_{0}, w_{1}, \ldots, w_{d-3}, z_{0}, z_{1}, \ldots, z_{d-3}\right\}
$$

and

$$
\begin{aligned}
E\left(H_{n, d}\right)= & E\left(G_{n-2 d+4, d}\right) \cup\left\{w_{i} v_{i} \mid 0 \leq i \leq d-3\right\} \\
& \cup\left\{w_{0} v_{2 d-4}, w_{0} v_{2 d-3}, w_{d-3} v_{d-2}, w_{d-3} v_{d-1}\right\} \\
& \cup\left\{w_{i} w_{i-1} \mid 0 \leq i \leq d-4\right\} \cup\left\{z_{i} u_{d-1+i} \mid 0 \leq i \leq d-3\right\} \\
& \cup\left\{z_{0} u_{d-3}, z_{0} u_{d-2}, z_{d-3} u_{2 d-3}, z_{d-3} u_{0}\right\} \\
& \cup\left\{z_{i} z_{i+1} \mid 0 \leq i \leq d-4\right\} .
\end{aligned}
$$

In Figure 3 we have depicted the graphs $G_{14,4}, G_{16,4}, G_{18,4}, H_{28.6}$, and $H_{34,6}$. 
238 JOURNAL OF GRAPH THEORY

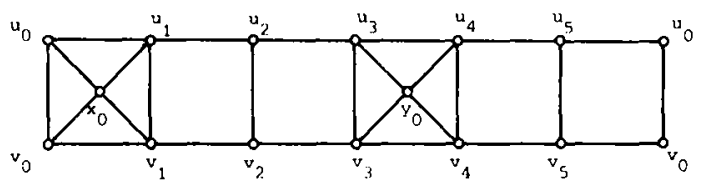

$G_{11,4}$

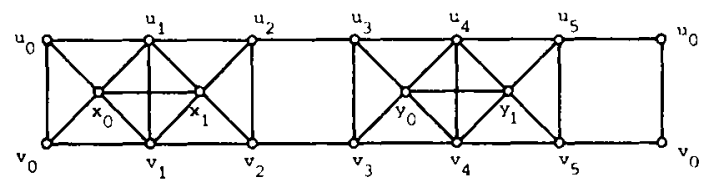

${ }_{16,4}$

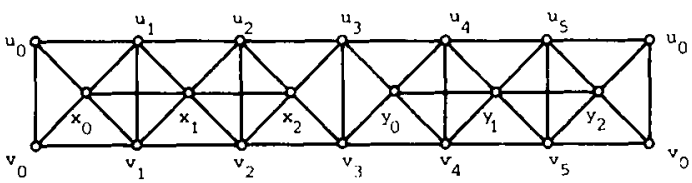

$G_{18,4}$
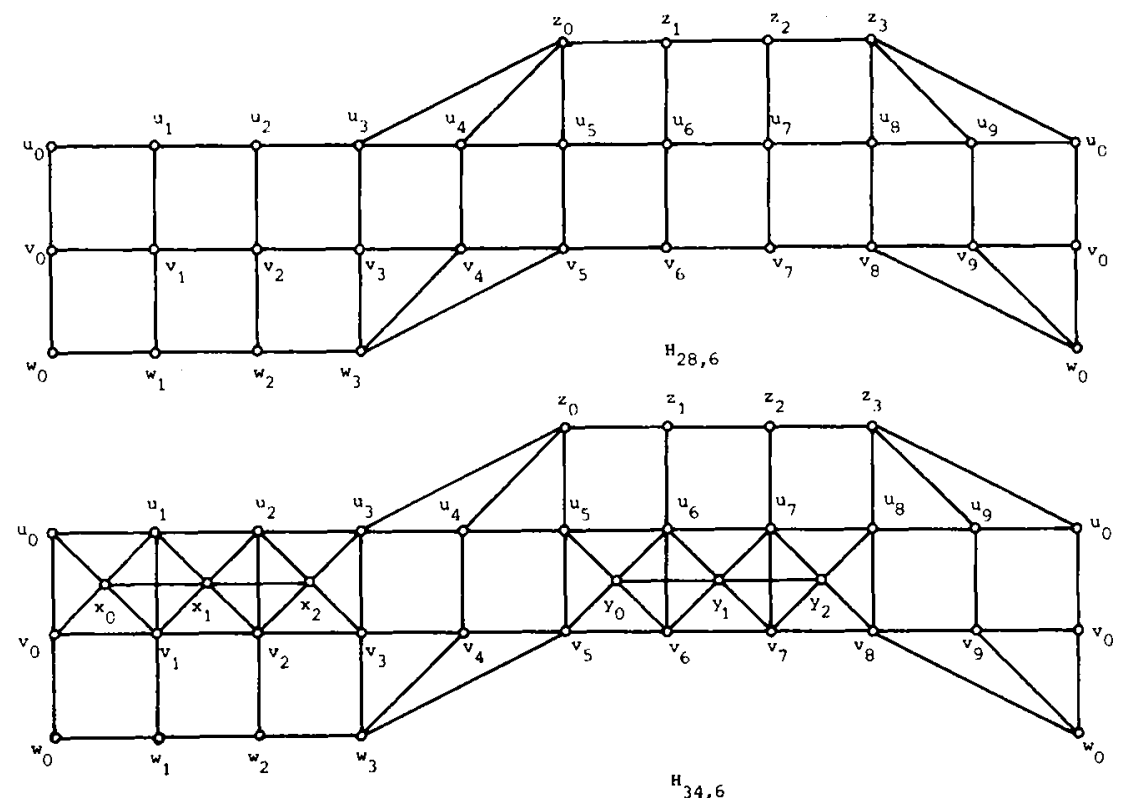

Fig. 3 


\section{References}

[1] M. Behzad, G. Chartrand, and L. Lesniak-Foster, Graphs and Digraphs. Prindle, Weber \& Schmidt, Boston, MA (1979).

[2] H. M. Mulder, $n$-Cubes and median graphs. J. Graph Theory 4 (1980) $107-110$.

[3] K. R. Parthasarathy and R. Nandakumar, Unique eccentric point graphs. Discrete Math. 46 (1983) 69-74. 